\begin{tabular}{|c|l|}
\hline Title & Anchoring protein crystals to mounting loops with hydrogel using inkjet technology \\
\hline Author(s) & Shinoda, A kira; Tanaka, Y oshikazu; Y ao, Min; Tanaka, Isao \\
\hline Citation & $\begin{array}{l}\text { Acta crystall lographica section D : biological crystall lography, 70(11), 2794 2799 } \\
\text { https://doi.org/10.1107/S139900471401476X }\end{array}$ \\
\hline Issue Date & 201411 \\
\hline Doc URL & http://hdl.handle.net/2115/57793 \\
\hline Type & article \\
\hline File Information & sendreprint-3.pdf \\
\hline
\end{tabular}

Instructions for use 


\title{
Anchoring protein crystals to mounting loops with hydrogel using inkjet technology
}

\author{
Akira Shinoda, Yoshikazu Tanaka, Min Yao and Isao Tanaka
}

Acta Cryst. (2014). D70, 2794-2799

Copyright (C) International Union of Crystallography

Author(s) of this paper may load this reprint on their own web site or institutional repository provided that this cover page is retained. Republication of this article or its storage in electronic databases other than as specified above is not permitted without prior permission in writing from the IUCr.

For further information see http://journals.iucr.org/services/authorrights.html

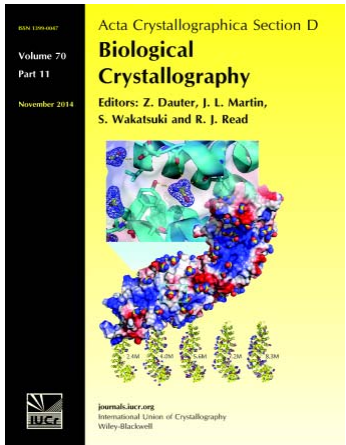

\begin{abstract}
Acta Crystallographica Section D: Biological Crystallography welcomes the submission of papers covering any aspect of structural biology, with a particular emphasis on the structures of biological macromolecules and the methods used to determine them. Reports on new protein structures are particularly encouraged, as are structure-function papers that could include crystallographic binding studies, or structural analysis of mutants or other modified forms of a known protein structure. The key criterion is that such papers should present new insights into biology, chemistry or structure. Papers on crystallographic methods should be oriented towards biological crystallography, and may include new approaches to any aspect of structure determination or analysis. Papers on the crystallization of biological molecules will be accepted providing that these focus on new methods or other features that are of general importance or applicability.
\end{abstract}

\section{Crystallography Journals Online is available from journals.iucr.org}


Acta Crystallographica Section D

Biological

Crystallography

ISSN 1399-0047

Akira Shinoda, ${ }^{a}$ Yoshikazu

Tanaka, ${ }^{\mathrm{a}, \mathrm{b}}$ Min $\mathrm{Yao}^{\mathrm{a}, \mathrm{b}}$ and

Isao Tanaka ${ }^{b_{*}}$

${ }^{a}$ Graduate School of Life Science, Hokkaido University, Sapporo, Hokkaido 060-0810,

Japan, and ${ }^{\mathbf{b}}$ Faculty of Advanced Life

Science, Hokkaido University, Sapporo,

Hokkaido 060-0810, Japan

Correspondence e-mail:

tanaka@castor.sci.hokudai.ac.jp

\section{Anchoring protein crystals to mounting loops with hydrogel using inkjet technology}

$\mathrm{X}$-ray crystallography is an important technique for structurebased drug discovery, mainly because it is the only technique that can reveal whether a ligand binds to the target protein as well as where and how it binds. However, ligand screening by $\mathrm{X}$-ray crystallography involves a crystal-soaking experiment, which is usually performed manually. Thus, the throughput is not satisfactory for screening large numbers of candidate ligands. In this study, a technique to anchor protein crystals to mounting loops by using gel and inkjet technology has been developed; the method allows soaking of the mounted crystals in ligand-containing solution. This new technique may assist in the design of a fully automated drug-screening pipeline.

\section{Introduction}

Structure-based drug discovery (SBDD) utilizes the threedimensional structure of target proteins for rational drug design and is expected to lead to the rapid development of drugs for diseases such as cancer, adult diseases and infectious diseases (Blundell et al., 2002). X-ray crystallography is an important technique for SBDD, and reveals not only whether the drug-candidate ligand binds to the protein but also where and how the ligand binds.

Crystallographic experiments for drug screening involve picking up a small crystal $(\sim 0.1 \mathrm{~mm})$ from a crystallization plate and soaking it in a solution containing ligand(s). After soaking, the crystal is removed from the solution and used for $\mathrm{X}$-ray data collection. Although data collection is typically performed within several minutes owing to the progress of synchrotron crystallography (Hiraki et al., 2008; Okazaki et al., 2008; Beteva et al., 2006; Fuchs, 2014; Soltis et al., 2008), ligand soaking is still performed manually. Therefore, the throughput is not satisfactory for screening a large number of candidate ligands. Thus, automated ligand soaking would be a powerful advance in the drug-screening pipeline.

In this study, we have developed a technique to anchor a $0.1 \mathrm{~mm}$-sized crystal to a crystal-mounting tool (or loop), which will allow treatment of the crystal using a robotic arm. Through automation of ligand soaking using a robot, fully automated ligand screening can be achieved, thereby accelerating drug screening. Furthermore, this technique can be applied to cryo-buffer screening, heavy-atom screening and dehydration experiments to improve crystal quality.
Received 8 May 2014 Accepted 23 June 2014

\section{Materials and methods}

\subsection{General strategy}

The ultimate aim of this study is to automate the process of drug screening by X-ray crystallography, which includes 
crystal recognition in the crystallization plate, anchoring of the crystal to a robotic arm, soaking of the crystal in ligandcontaining buffer and X-ray diffraction data collection. In the present study, we explored the possibility of anchoring the protein crystal to a robotic arm to automate the latter part of the experiments (ligand soaking and data collection). Technically, it would be feasible to handle protein crystals with a micro-manipulator (Khajepour et al., 2013). However, multiple crystals need to be soaked simultaneously for rapid screening; thus, it is desirable to fix protein crystals to a small tool such as a crystal-mounting loop.

Anchoring of protein crystals to a crystal-mounting loop is challenging. Firstly, protein crystals are susceptible to environmental changes, and since they are composed of $\sim 50 \%$ water (by volume) they collapse quickly outside the solution. To prevent this, the crystal must be anchored to the loop and returned to the buffer solution within seconds. In our system, the position and shape of the crystal is recognized by a highspeed image-processing application and an $X Y$ stage moves the crystal to the right place quickly. In addition, protein crystals are susceptive to physical stress. To minimize chemical and physical stresses, we explored the use of hydrogel solutions ejected by inkjet technology on the surface of the protein crystal to anchor them to the mounting loop.

\subsection{Gels for anchoring protein crystals}

Gels are classified into several groups on the basis of the triggers for gelation, which include ions, radicals, heat and chemical bond formation. In this study, gels triggered by ions and chemical bond formation were evaluated. Gels triggered by ions are normally formed very quickly, while those triggered by chemical bonds are usually homogenous and of high quality. We used tetrapolyethylene glycol (tetra-PEG) gel, the main chain of which, PEG, is often used for protein crystallization. Tetra-PEG gel is prepared by a coupling reaction of two kinds of four-armed PEG monomers with different functional groups at the ends (Fig. 1; Sakai et al., 2008). Among several combinations, we found that the gel formed by maleimide-treated tetra-PEG [10\% $(w / v)$ PTE-100MA; Nichiyu, Japan] and thiol-treated tetra-PEG [10\%(w/v) PTE100SH; Nichiyu, Japan] was ideal as it forms quickly and is sufficiently strong. The reaction proceeds owing to the

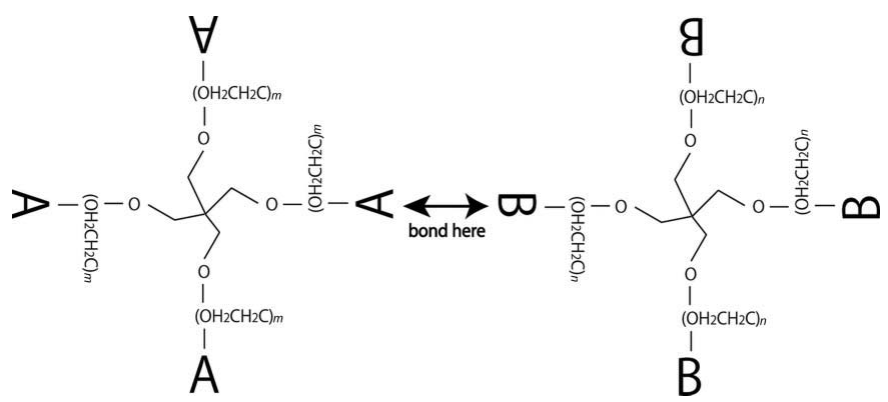

Figure 1

Tetra-PEG gel. A and B are functional groups that undergo a crosslinking coupling reaction. We used gel solutions of maleimide (A) and thiol (B). The chain lengths $m$ and $n$ were both 100 . nucleophilic attack of the thiolate anions (Michael addition reaction) and is catalyzed by a base. The gelation speed is further enhanced by using an inkjet device since the mixing of tiny drops is quick. The gelation time is less than $1 \mathrm{~s}$ under these conditions, which allows the crystal to be anchored quickly, minimizing stress on the crystal.

\subsection{System for anchoring protein crystals}

The system is composed of an inkjet device (IJK-200H, Microjet, Japan) with two nozzles (IJHD-100 and IJHD-300), $X Y Z$ stages for moving crystals and nozzles, a rotation motor for crystals, a microscopic CCD camera and an operating computer (Fig. 2). The motor and stages are controlled by a computer through an RS232 cable via an electronic circuit operated by a PIC microchip IC. The main program is coded using Microsoft Visual Studio Express C\#. The images from the microscopic CCD camera are sent to the computer and captured with DirectShow wrapped by DirectShow-lib2005. Image processing is based on a well studied computed tomography algorithm (Whitaker \& Elangovan, 2002). This process is accelerated using the CUDA parallel computing platform of NVIDIA. The image-processing program is compiled as a dynamic-link library with Microsoft Visual Studio Express $\mathrm{C}++$ and CUDA SDK and then used by the main program. The system automatically recognizes the crystal shape in three dimensions, displays it on the monitor of the main computer using DirectX, decides where to eject gel to anchor the crystal and operates the motors and inkjet device. The total time required is about $6 \mathrm{~s}$ ( $3 \mathrm{~s}$ for recognizing the crystal shape and $3 \mathrm{~s}$ for ejecting the gel).

The distance between the inkjet exit and the crystal is set to approximately $10 \mathrm{~mm}$. This distance is long enough to avoid accidental mixing of the two gel solutions at the tip of the inkjet head and sealing of the head, and is sufficiently short to avoid significant slowing of the ejection of the gel solution

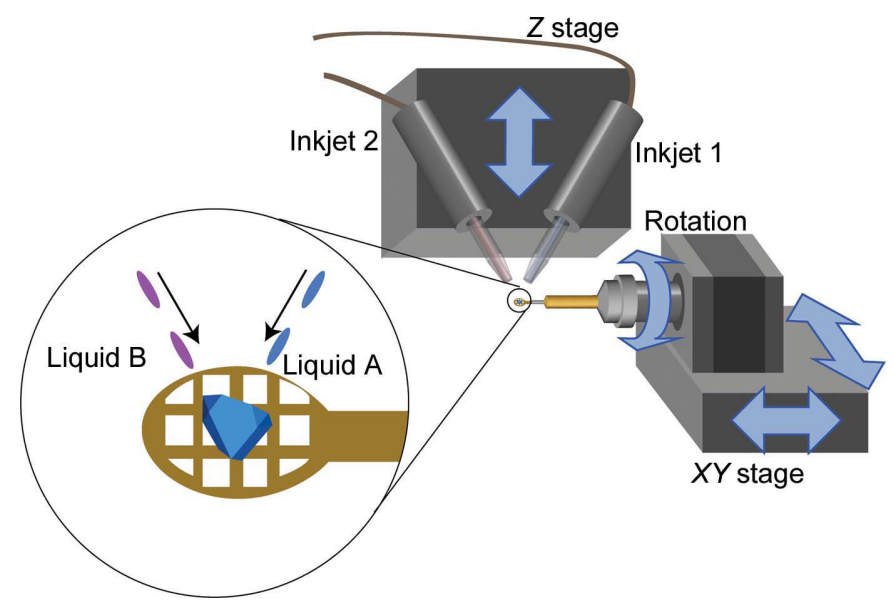

Figure 2

System for anchoring protein crystals. The crystal-mounting loop (encircled) is attached to a spindle directly connected to the rotation motor. The crystal can be moved in the horizontal plane using the $X Y$ stage. Two inkjet nozzles are connected to the $Z$ stage and can move in the vertical plane. 
Table 1

Summary of crystallization conditions, data-collection and refinement statistics.

Values in parentheses are for the highest resolution shell.

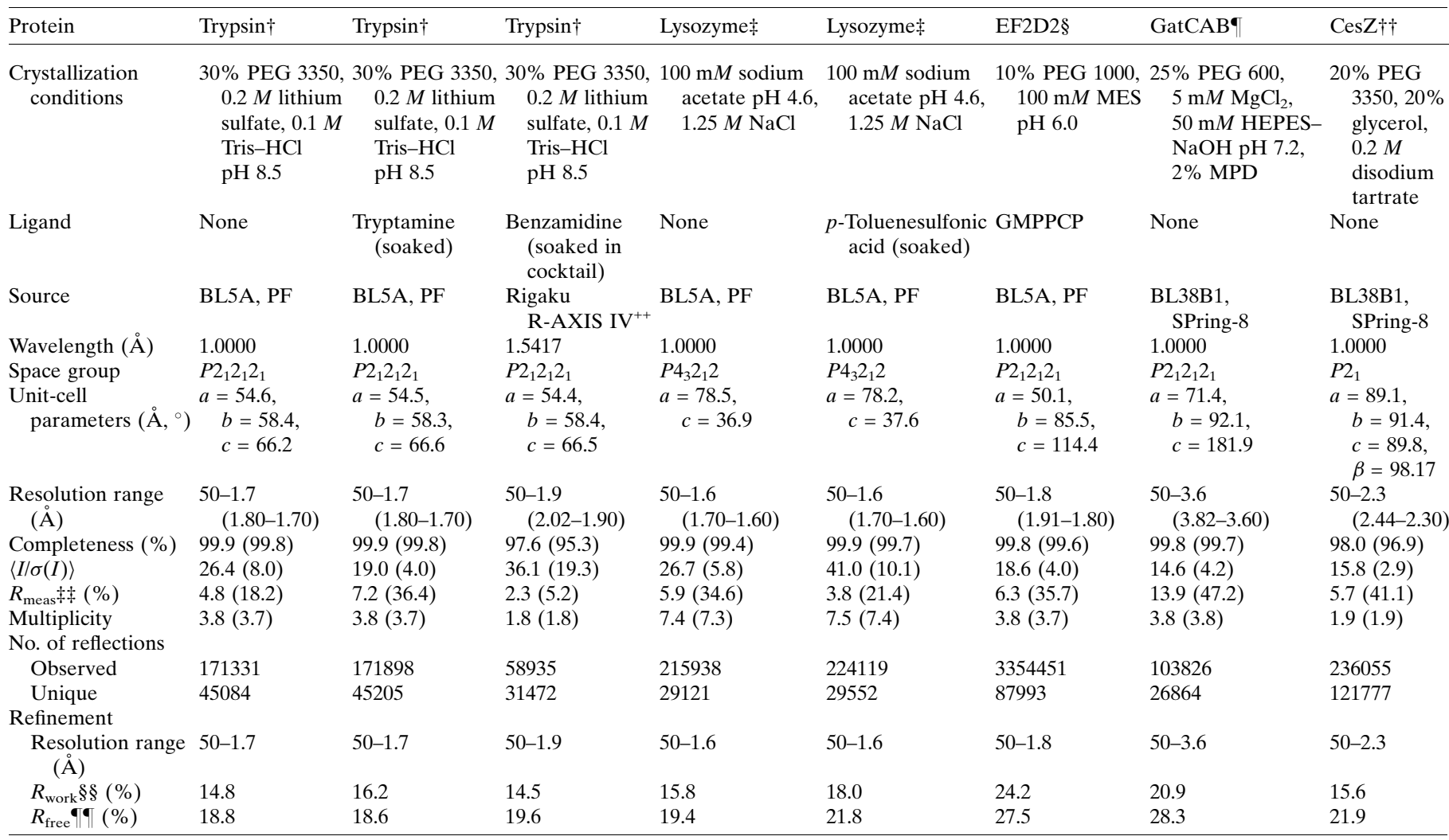

$\dagger$ Bovine pancreatic trypsin. $\ddagger$ Hen egg-white lysozyme. \& Translation elongation factor EF2 domain I-II from Pyrococcus horikoshii. 9 GatCAB from Staphylococcus aureus

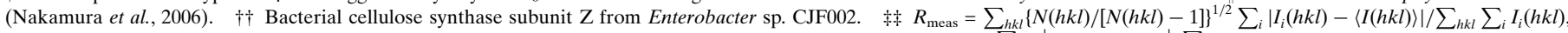

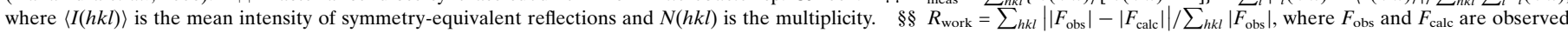
and calculated structure-factor amplitudes, respectively. The $R_{\text {free }}$ value was calculated as for $R_{\text {work }}$ but using only an unrefined subset of reflection data.

( $\left.\sim 6 \mathrm{~m} \mathrm{~s}^{-1}\right)$ by air friction or drifting of the solution owing to the air flow in the room.

\subsection{Processes for anchoring protein crystals}

Immediately after mounting the crystal on the device, images are captured by the CCD camera while the crystal rotates $360^{\circ}$ and a three-dimensional image of the crystal is created. The crystal is then rotated to the most suitable angle for ejecting drops of the gel solution. Using the inkjet, it is possible to eject small amounts of gel solution without excess physical stress. We used IJHD-100 and IJHD-300 inkjet heads for ejecting $10 \%(w / v)$ PTE-100MA containing $2 \mathrm{mM}$ tetramethylethylenediamine (TEMED) and 10\%( $w / v)$ PTE-100SH solutions, respectively. The inkjet ejects a few drops of gel solution when a pulse signal is generated. For each target point, gel solutions corresponding to three pulses $(60 \mathrm{~V}$ and $70 \mu$ s width) for IJHD-100 and one pulse $(60 \mathrm{~V}$ and $130 \mu \mathrm{s}$ width) for IJHD-300 are ejected. The total amounts are $200 \mathrm{pl}$ per pulse for IJHD-100 and $190 \mathrm{pl}$ per pulse for IJHD-300 (Fig. 3).

For mounting crystals, we used mesh-type LithoLoops of $0.40 \mathrm{~mm}$ diameter (Protein Wave Co.) attached to a $16 \mathrm{~mm}$ CrystalCap Copper Magnetic base (Hampton Research). A meshed loop was more stable than a nylon loop for anchoring crystals. After ejecting the gel solution, a thin layer of the gel

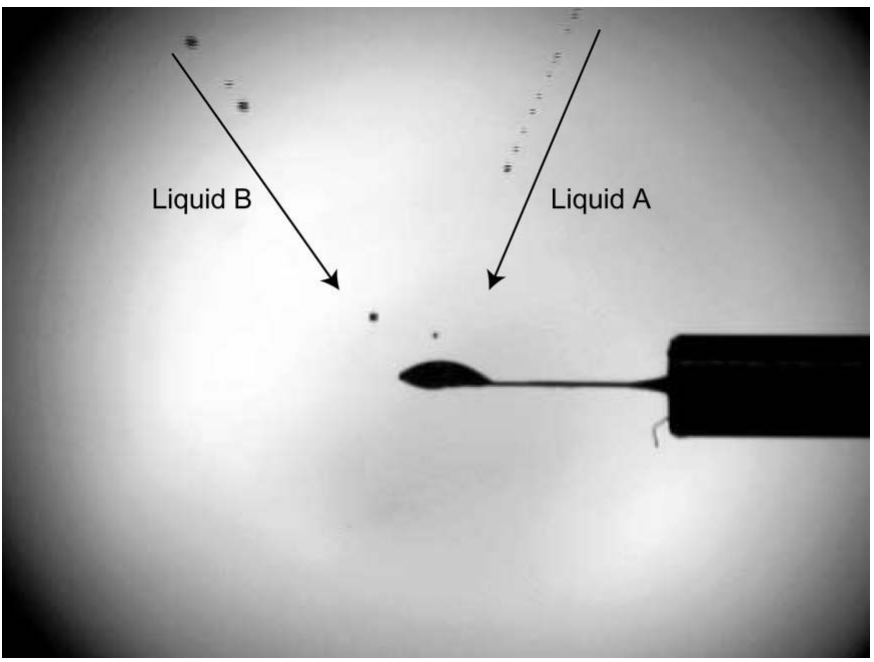

Figure 3

Ejection of tetra-PEG gel solutions. This picture was taken using strobelighting technology with a microscopic CCD camera. Liquid $\mathrm{A}$ is $10 \%(w / v)$ PTE-100MA containing $2 \mathrm{~m} M$ TEMED ejected by an IJHD100 inkjet head and liquid B is PTE-100SH ejected by an IJHD-300 inkjet head. 


\section{research papers}

fixes the crystal to the loop. The gel layer is so thin that X-ray absorption is negligible (Fig. 4).

The gel solutions are aspirated from the head of the inkjet nozzles. The advantages of aspiration from the nozzle head over that from the bottom are as follows: (i) the inkjet is not stacked, which implies that dust and other foreign particles will be blocked because the tip of the inkjet head is smaller than the inside, (ii) air bubbles are avoided by using $5 \mathrm{kPa}$ to aspirate $150 \mu \mathrm{l}$ gel solution over 20-40 min and (iii) less gel is used: only $1 \mu l$ is needed to anchor a crystal and, by aspirating from the head, $100 \mu \mathrm{l}$ is used to fill the device, which compares favourably with $1000 \mu \mathrm{l}$ when filled from the bottom.

The gel solution at the tip of the inkjet nozzle is slowly concentrated by gradual evaporation, which could lead to stacking. To avoid this, the system automatically recognizes whether the crystal is mounted, and if it is not mounted it discards the gel solution every $30 \mathrm{~s}$. The system also discards the gel solution corresponding to 50 pulses just before starting the ejection. This stabilizes the inkjet and enables the ejection of precisely the right amount at the right place.

\section{Results}

The proposed system was evaluated by applying it to five protein crystals obtained at a $\mathrm{pH}$ ranging from 4.5 to 8.5 and using a variety of precipitants such as $1.25 \mathrm{M} \mathrm{NaCl}$ or $30 \%$ PEG 3350, as listed in Table 1. The effect of the gel coating on each crystal was inspected visually and by X-ray diffraction. Two crystals (lysozyme and trypsin) were further soaked in solutions containing known ligands.

Crystals were anchored to the loop using the proposed system; they were then kept in stabilization buffer in our laboratory and transported to the Photon Factory (PF), Tsukuba, Japan or SPring-8, Hyogo, Japan for X-ray diffraction experiments. Prior to X-ray diffraction, the crystals were soaked in their respective cryobuffers with or without ligand and flash-cooled in a cryostream at $100 \mathrm{~K}$. The diffraction data were processed and scaled with XDS (Kabsch, 2010). Molecular replacement and refinement were performed with PHENIX (Adams, 2010).

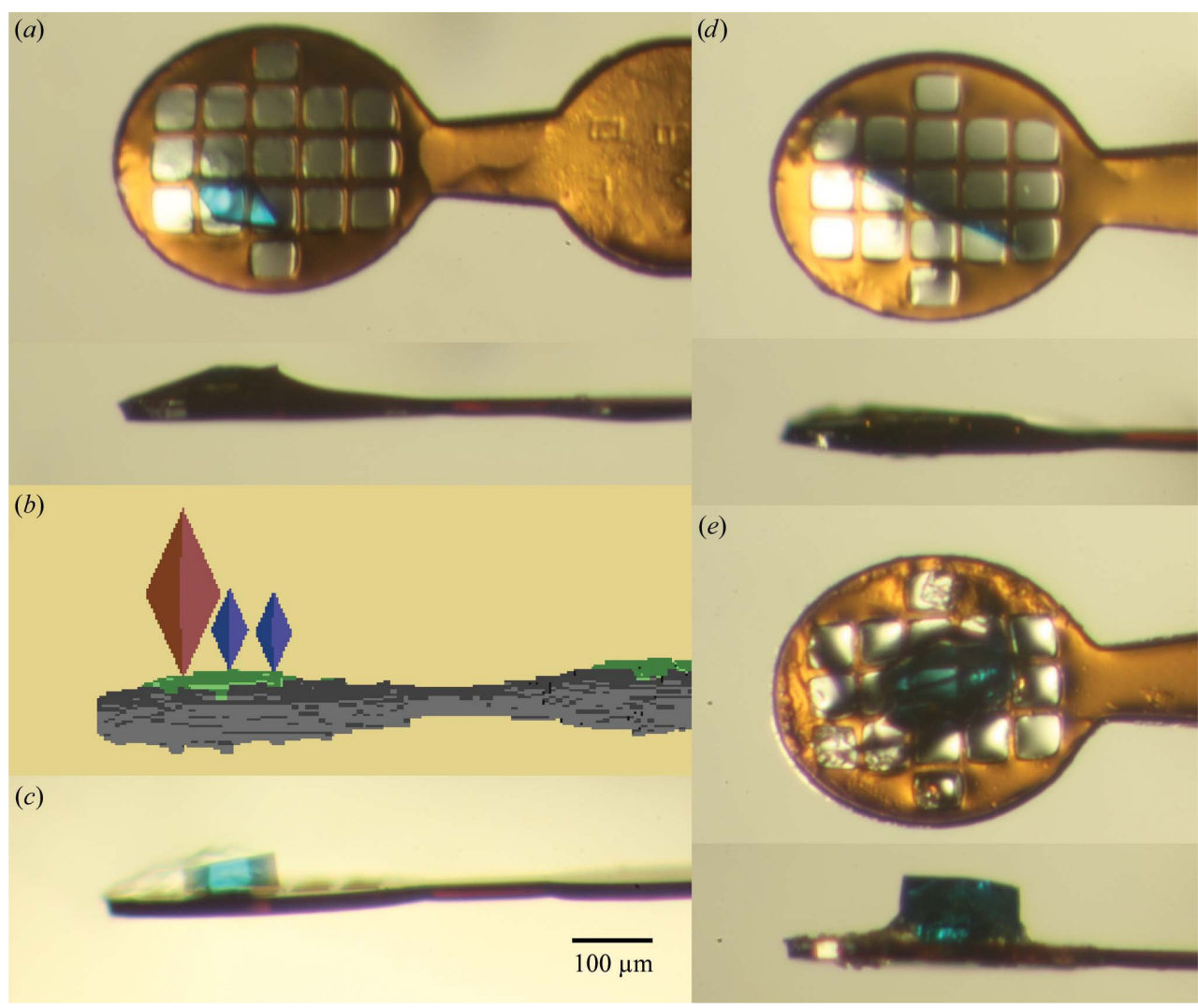

Microscopic images of crystals anchored to mesh-type LithoLoops (Protein Wave Co.). All crystals are dyed with Izit dye (Hampton Research). (a) Top and side views of an anchored CesZ crystal. (b) The 列 stabilization buffer. The thin layer around the crystal is gel. $(d)$ Top and side views of an anchored GatCAB crystal. (e) Top and side views of an anchored lysozyme crystal.

The same procedures were used to anchor all crystals to the loop. That is, approximately $0.8 \mathrm{nl}(0.2 \mathrm{nl} \times 3+0.19 \mathrm{nl} \times 1)$ gel solution was ejected on each target point aligned at intervals of seven pixels $(\sim 67 \mu \mathrm{m})$ at the centre of the crystal (Fig. $4 c)$. Immediately after being anchored to the loop by the gel, the crystal along with the loop is placed in a container filled with stabilization solution. The remaining gel solution on the crystal or within the gel is diluted by the stabilization solution, which will reduce any adverse effects on the crystal.

Relatively large quantities of gel solutions were applied to ensure the anchoring of the lysozyme crystal (discussed later). In spite of this, no negative influence was observed on visual inspection (Fig. 4) or by X-ray diffraction. No significant change in resolution was detected and all crystals were therefore suitable for ligand-screening experiments.

For two proteins (trypsin and lysozyme), anchored crystals were soaked with known ligands $(100 \mathrm{~m} M$ tryptamine for trypsin and $100 \mathrm{~m} M p$-toluenesulfonic acid for lysozyme) for a fixed time (20 min for trypsin and 8 min for lysozyme) and they were directly transferred to the X-ray diffraction equipment for data collection. Although 10\% DMSO was contained in the soaking buffer for one of the ligands (tryptamine), the gel was not affected. We have also performed a cocktail 
experiment using buffer containing five ligands ( $2 \mathrm{~m} M$ tryptamine, $2 \mathrm{~m} M$ 4-aminobenzoic acid, $2 \mathrm{~m} M$ benzamidine, $2 \mathrm{~m} M$ $p$-toluenesulfonic acid and $2 \mathrm{~m} M$ 1,4,8,11-tetraazacyclotetradecane). The conditions for data collection and refinement are shown in Table 1 . The $F_{\mathrm{o}}-F_{\mathrm{c}}$ map calculated after refinement clearly showed the bound ligand in the appropriate binding site (Fig. 5). Thus, the gel used for anchoring had no adverse effect on ligand binding.

\section{Discussion}

Protein crystals contain internal solvent, which typically amounts to $\sim 50 \%$ of the volume of the crystal (Matthews, 1968) and makes them very fragile and difficult to handle. To measure X-ray diffraction data, protein crystals are usually mounted on a tiny nylon loop attached to the top of a pin. The crystal is retained within the loop owing to the surface tension of water until it is frozen. Clearly, this method cannot be used for experiments in solution, which are necessary to evaluate ligand binding. In the present work, we show that protein crystals can be fixed to the loop using gel and can subsequently be used for soaking experiments.

We first examined suitable materials to fix protein crystals. To perform ligand-soaking experiments, this material should not interfere with permeation of protein crystallization buffer or ligands. A hydrophilic gel is best suited for this purpose. However, crystals may be damaged when the gel solution contacts the crystal before gelation is complete. Therefore, we screened hydrophilic gel solutions (such as agarose gel, acrylamide gel, hyaluronic acid gel and silicone hydrogel) that can instantly gelate in order to identify those most suitable for anchoring protein crystals.

Sodium alginate is known to gelate very quickly: within $1 \mathrm{~s}$ of the introduction of concentrated calcium ions. However, the requirement of a high concentration of calcium ions limits the use of this gel. Even after gelation, the gel would easily collapse if exposed to solutions of low ion concentration. Furthermore, to fix crystals with sufficient strength using sodium alginate gel, the concentration of the gel solution has to be greater than $1 \%(w / v)$. However, sodium alginate solution at this concentration has a viscosity of greater than $80 \mathrm{mPa}$, which is higher than the limit that the inkjet we use can eject
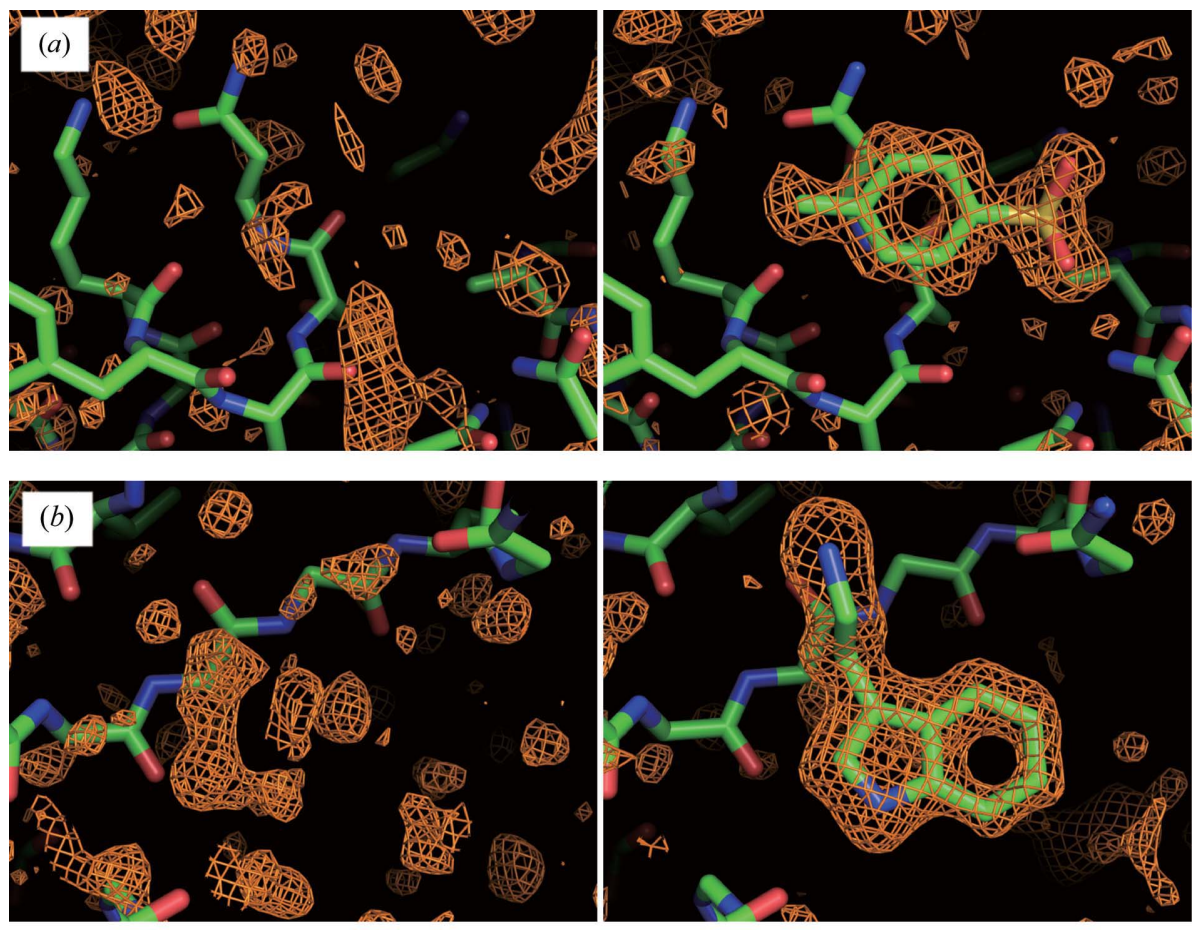

Figure 5

$F_{\mathrm{o}}-F_{\mathrm{c}}$ maps (contoured at the $2 \sigma$ level) around the ligand-binding sites of gel-anchored crystals. (a) Lysozyme with (right) and without (left) ligand soaking. (b) Trypsin with ligand (tryptamine) soaking (right) and without ligand soaking (left). (c) Trypsin soaked in the cocktail solution containing five ligands (2 $\mathrm{m} M$ tryptamine, $2 \mathrm{~m} M$ 4-aminobenzoic acid, $2 \mathrm{~m} M$ benzamidine, $2 \mathrm{~m} M$ p-toluenesulfonic acid and $2 \mathrm{~m} M$ 1,4,8,11-tetraazacyclotetradecane). The electron density clearly shows that benzamidine is bound. The map was drawn using Coot (Emsley \& Cowtan, 2004).

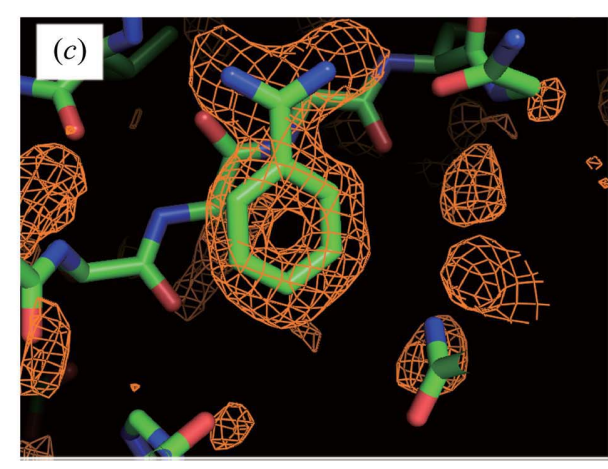
(40 $\mathrm{mPa}$ s). Moreover, sodium alginate forms aggregated materials when mixed with other polymers such as PEG, which severely limits the usable materials for crystallization. For these reasons, sodium alginate gel was not utilized.

To hold a crystal in a solution, the gel needs to be physically strong. At the same time, thick gel layers must be avoided because they will absorb X-rays and decrease the data quality. More concentrated gel solutions or gels with longer chains must be used to anchor crystals using a small amount of gel solution with sufficient strength. However, this leads to increased viscosity of the gel solution and makes it difficult to handle (especially in automated ejection). Thus, a gel with properties that meet mutually conflicting demands, i.e. high gelation speed and physical strength as well as low viscosity of the gel solution, is required. We found that the tetra-PEG gel meets these conditions.

There are two ways to place gel solution on a crystal: using a contacttype or a non-contact-type machine. Using a dispensing machine such as a micro-injector, a contact-type machine, the gel solution is directly placed. However, direct contact has the risk of physically damaging the crystal and it is difficult to handle such small amounts of 
liquid. Non-contact-type machines can avoid these problems. Therefore, we examined sprays and inkjets. For anchoring small crystals using the least amount of gel solution, the inkjet is more suitable.

The inkjet heads used were IJHD-100 and IJHD-300 from Microjet Corporation, Japan. These inkjet heads can eject liquid with a relatively high viscosity of up to $\sim 40 \mathrm{mPa}$. However, since the viscosity of gel solutions is usually very high, our first attempt failed. There are two ways to lower the viscosity of the gel solution. One is to lower the concentration and the other is to shorten the chain length of the gel molecules. However, both methods decrease the gelation speed. In other words, the gelation speed would be too slow for the solution condition that the inkjet could eject. This problem was solved using amine catalysis. The gelation reaction based on Michael addition can be accelerated by amine catalysis. Thus, $2 \mathrm{~m} M$ TEMED was added to one of the gel solutions $[10 \%(w / v)$ PTE-100MA] and as a result the gelation time was dramatically shortened. It is also worth mentioning that use of an inkjet accelerates the gelation speed because the tiny drops ejected allow more efficient mixing of the two solutions. In fact, gelation is completed in less than $1 \mathrm{~s}$ when both catalysis and an inkjet are used. We used this rapid gelation technique for anchoring crystals.

For crystals in acidic conditions, the gelation speed is lower. The lysozyme crystal at $\mathrm{pH} 4.5$ required the maximum amount of gel solutions to anchor. The protocol was adjusted for this case such that the gel solutions collide with each other directly and gelate without touching the crystal. However, other solutions remain ungelated before the crystal is transferred and the solutions were washed away in stabilization solution. This situation can be improved by ejecting gel solutions more accurately and mixing them together before diffusion in crystallization buffer on the crystal surface.

Protein crystals easily collapse when exposed to air; thus, the crystal must be handled quickly outside the solution. Crystal recognition by image processing is accelerated using GPGPU (general-purpose computing on graphics processing units). Crystal centring, which usually takes time, is not needed for this system. Instead, the system automatically recognizes the crystal and rotates it to the best angle, and the $X Y Z$ stages move the crystal and the inkjet nozzles to the best positions for ejecting gel solution. This automation enables anchoring of crystals without damage. High-speed ALS-250C2P stages (Chuo Precision Industrial, maximum speed $\left.16 \mathrm{~mm} \mathrm{~s}^{-1}\right)$ with high moving resolution $(2 \mu \mathrm{m})$ and an electronic circuit specifically made for this purpose enable the system to complete all crystal movement in only a few seconds.

Using our proposed technique, soaking of protein crystals, which is usually performed manually by highly skilled persons, can be automated, thereby accelerating ligand screening. Such an automation system should consist of three units, a 'crystal anchoring unit', a 'crystal soaking unit' and a 'crystal freezing unit'. Once the crystal is anchored to the loop, the latter two units can be built straightforwardly. Furthermore, once the crystal is anchored to a tool, it would be possible to manage the experiments within preset timeframes; for example, soaking experiments for a few seconds. Therefore, analysis of the effect of the soaking time is possible. Finally, experiments that require repeated soaking, such as in-crystal chemical ligation (Yamane et al., 2010), would also become feasible.

We would like to thank Dr Takayuki Kurokawa and Dr Jian Ping Gong, Hokkaido University for very important and helpful suggestions. We are also grateful to the beamline staff of Photon Factory and SPring-8 for their help in collecting $\mathrm{X}$-ray diffraction data. This work was supported by a Grant-inAid for Challenging Exploratory Research from the Ministry of Education, Culture, Sports, Science and Technology of Japan (No. 25650015).

\section{References}

Adams, P. D. et al. (2010). Acta Cryst. D66, 213-221.

Beteva, A. et al. (2006). Acta Cryst. D62, 1162-1169.

Blundell, T. L., Jhoti, H. \& Abell, C. (2002). Nature Rev. Drug Discov. 1, 45-54.

Emsley, P. \& Cowtan, K. (2004). Acta Cryst. D60, 2126-2132.

Fuchs, M. R. et al. (2014). J. Synchrotron Rad. 21, 340-351.

Heidari Khajepour, M. Y., Vernede, X., Cobessi, D., Lebrette, H., Rogues, P., Terrien, M., Berzin, C. \& Ferrer, J.-L. (2013). Acta Cryst. D69, 381-387.

Hiraki, M., Watanabe, S., Honda, N., Yamada, Y., Matsugaki, N., Igarashi, N., Gaponov, Y. \& Wakatsuki, S. (2008). J. Synchrotron Rad. 15, 300-303.

Kabsch, W. (2010). Acta Cryst. D66, 125-132.

Matthews, B. W. (1968). J. Mol. Biol. 33, 491-497.

Nakamura, A., Yao, M., Chimnaronk, S., Sakai, N. \& Tanaka, I. (2006). Science, 312, 1954-1958.

Okazaki, N., Hasegawa, K., Ueno, G., Murakami, H., Kumasaka, T. \& Yamamoto, M. (2008). J. Synchrotron Rad. 15, 288-291.

Sakai, T., Matsunaga, T., Yamamoto, Y., Ito, C., Yoshida, R., Suzuki, S., Sasaki, N., Shibayama, M. \& Chung, U. (2008). Macromolecules, 41, 5379-5384.

Soltis, S. M. et al. (2008). Acta Cryst. D64, 1210-1221.

Whitaker, R. T. \& Elangovan, V. (2002). Med. Image Anal. 6, 235-249.

Yamane, J., Ohyabu, N., Yao, M., Takemoto, H. \& Tanaka, I. (2010). J. Appl. Cryst. 43, 1329-1337. 